Article

\title{
Fixed Point Results for F-Contractions in Cone Metric Spaces over Topological Modules and Applications to Integral Equations
}

\author{
Adrian Nicolae Branga (D)
}

Department of Mathematics and Informatics, Lucian Blaga University of Sibiu, Dr. I. Raţiu Street, No. 5-7, 550012 Sibiu, Romania; adrian.branga@gmail.com or adrian.branga@ulbsibiu.ro

\begin{abstract}
In this paper, the concept of $F$-contraction was generalized for cone metric spaces over topological left modules and some fixed point results were obtained for self-mappings satisfying a contractive condition of this type. Some applications of the main result to the study of the existence and uniqueness of the solutions for certain types of integral equations were presented in the last part of the article, one of them being a fractional integral equation.
\end{abstract}

Keywords: F-contractions; fixed point theorems; integral equations; topological left modules; cone metric spaces

\section{Introduction}

The concept of cone metric space was introduced by Huang and Zhang [1] as a generalization of a metric space, proving that Banach's contraction theorem remains valid in this context. Afterwards, many authors have obtained fixed point results on cone metric spaces: Radenović and Rhoades [2], Rezapour and Hamlbarani [3], Kadelburg et al. [4], Du [5] and the references therein. Further, Liu and Xu [6] introduced the concept of cone metric space over a Banach algebra and proved some fixed point theorems for Lipschitz mappings. Later on, $\mathrm{Xu}$ and Radenović [7] extended the results of Liu and Xu [6], without the assumption of normality of the cone involved. Also, generalizations of Banach's theorem have been obtained in other directions. Wardowski [8] defined the class of F-contractions and proved a fixed point result as a extension of Banach contraction principle. After this, Wardowski and Van Dung [9] introduced the concept of $F$-weak contraction and obtained a new fixed point theorem. Cosentino and Vetro [10] obtained new fixed point theorems of Hardy-Rogers type for F-contractions in ordered metric spaces. Other results concerning F-contractions have been obtained by: Piri and Kumam [11], Minak et al. [12], Ahmad et al. [13], Kadelburg and Radenović [14], Dey et al. [15], Wardowski [16], Alfaqih et al. [17], Karapinar et al. [18] and the references therein.

Regarding the fractional differentiation and integration, many models have been proposed in the literature: the Riemann-Liouville fractional model [19], the Caputo model [20], the Atangana-Baleanu (or AB) fractional model [21,22], the generalised proportional fractional (or GPF) model [23], the Prabhakar fractional model [24,25], and others. Fernandez et al. [26] proposed a unified model of fractional calculus by using a general operator which includes many types of fractional operators. They consider some fractional differential equations and solve a general Cauchy problem in this new framework. Important results on nonlinear fractional differential equations were obtained by Agarwal et al. [27,28], SoradiZeid et al. [29], Almeida [30], Khan et al. [31], Keten et al. [32] and the references therein.

In this article, we generalize the notion of cone metric space for topological left module and we define the concept of F-contraction on this new space. Next, we obtain some fixed point results for self-mappings satisfying a contractive condition of this type. In the last part of the article, some applications of the main result to the study of the existence and 
uniqueness of the solutions for integral equations were presented, one of them being an integral equation of fractional type.

\section{Methods}

We will start by presenting some notions relating to ordered topological rings and topological left modules. For more details see the papers Warner [33].

Definition 1. A ring $(\mathcal{A}, \oplus, \odot)$ is called a topological ring if the set $\mathcal{A}$ is endowed with a topology $\tau_{\mathcal{A}}$ such that the mappings $\oplus: \mathcal{A} \times \mathcal{A} \rightarrow \mathcal{A}, \ominus: \mathcal{A} \rightarrow \mathcal{A}, \odot: \mathcal{A} \times \mathcal{A} \rightarrow \mathcal{A}$ are continuous, where $\mathcal{A} \times \mathcal{A}$ is considered with respect to the product topology.

We denote by $\left(\mathcal{A}, \oplus, \odot, \tau_{\mathcal{A}}\right)$ a topological ring.

Definition 2. Let $(\mathcal{A}, \oplus, \odot)$ be a ring and let $\preceq$ be a partial order relation on $\mathcal{A}$. $\mathcal{A}$ is called a partially ordered ring if it fulfills the following conditions:

(i) if $a, b, c \in \mathcal{A}, a \preceq b$, then $a \oplus c \preceq b \oplus c$;

(ii) if $a, b \in \mathcal{A}, 0 \preceq a, 0 \preceq b$, then $0 \preceq a \odot b$.

We denote by $(\mathcal{A}, \oplus, \odot, \preceq)$ a partially ordered ring.

The set $\mathcal{A}_{+}=\left\{a \in \mathcal{A} \mid 0_{\mathcal{A}} \preceq a\right\}$ is named the positive cone of $\mathcal{A}$.

A partially ordered topological ring and is denoted by $\left(\mathcal{A}, \oplus, \odot, \tau_{\mathcal{A}}, \preceq\right)$.

Definition 3. Let $(\mathcal{A}, \oplus, \odot)$ be a ring. A left $\mathcal{A}$-module consists of an abelian group $(E,+)$ and a mapping $\cdot: \mathcal{A} \times E \rightarrow$ E satisfying the following properties:

(1) if $a \in \mathcal{A}, x, y \in E$, then $a \cdot(x+y)=a \cdot x+a \cdot y$;

(2) if $a, b \in \mathcal{A}, x \in E$, then $(a \oplus b) \cdot x=a \cdot x+b \cdot x$;

(3) if $a, b \in \mathcal{A}, x \in E$, then $(a \odot b) \cdot x=a \cdot(b \cdot x)$;

(4) if $x \in E$, then $1_{\mathcal{A}} \cdot x=x$.

A left $\mathcal{A}$-module is denoted by $(E,+, \cdot)$.

Definition 4. Let $\left(\mathcal{A}, \oplus, \odot, \tau_{\mathcal{A}}\right)$ be a topological ring. A left $A$-module $(E,+, \cdot)$ is called a topological left $A$-module if on $E$ is specified a topology $\tau_{E}$ such that the mappings $+: E \times E \rightarrow E$, $-: E \rightarrow E, \cdot: \mathcal{A} \times E \rightarrow E$ are continuous, where $E \times E$ and $\mathcal{A} \times E$ are considered with respect to the product topologies, respectively.

A topological left $\mathcal{A}$-module is denoted by $\left(E,+, \cdot, \tau_{E}\right)$.

A topological left $\mathcal{A}$-module, $\left(E,+, \cdot, \tau_{E}\right)$, where $\tau_{E}$ is a Hausdorff topology, is called a Hausdorff topological left $\mathcal{A}$-module.

\section{Results}

Definition 5. Let $\left(\mathcal{A}, \oplus, \odot, \tau_{\mathcal{A}}, \preceq\right)$ be a partially ordered topological ring and $\left(E,+, \cdot, \tau_{E}\right)$ a topological left $\mathcal{A}$-module. A cone is a non-empty subset $P$ of E satisfying the conditions:

(1) $P \neq\left\{0_{E}\right\}$ and $P \cap(-P)=\left\{0_{E}\right\}$;

(2) $P$ is closed;

(3) $a \cdot x+b \cdot y \in P$ for all $a, b \in \mathcal{A}_{+}, x, y \in P$.

The cone $P$ is solid if int $(P) \neq \varnothing$.

We define the set $P^{*}=P \backslash\left\{0_{E}\right\}$.

Lemma 1. Let $\left(\mathcal{A}, \oplus, \odot, \tau_{\mathcal{A}}, \preceq\right)$ be a partially ordered topological ring, with $1_{\mathcal{A}} \in \mathcal{A}_{+},\left(E,+, \cdot, \tau_{E}\right)$ a topological left $\mathcal{A}$-module and $P$ a solid cone of $E$. Then, the following statements are valid:

(i) $P+P \subseteq P$;

(ii) the relation $\leq_{P}$ over $E$, defined by $x \leq_{P} y$ if and only if $y-x \in P$, is a partial order relation on $E$;

(iii) if $x \leq_{P} y$ and $y \ll_{P} z$, then $x \ll_{P} z$, for all $x, y, z \in E$;

(iv) if $x \leq_{P} y$ and $u \leq_{P} v$, then $x+u \leq_{P} y+v$, for all $x, y, u, v \in E$. 
Lemma 2. Let $\left(\mathcal{A}, \oplus, \odot, \tau_{\mathcal{A}}, \preceq\right)$ be a partially ordered topological ring, $\left(E,+, \cdot, \tau_{E}\right)$ a topological left $\mathcal{A}$-module and $P$ a solid cone of $E$. Then, the following statements are valid:

(i) $\quad \operatorname{int}(P) \cap(-P)= \begin{cases}\varnothing, & 0_{E} \notin \operatorname{int}(P) \\ \left\{0_{E}\right\} & , 0_{E} \in \operatorname{int}(P) ;\end{cases}$

(ii) $\operatorname{int}(P) \cap \operatorname{Fr}(P)=\varnothing$;

(iii) assuming that $\tau_{\mathcal{A}}$ is a Hausdorff topology, $0_{\mathcal{A}}$ is an accumulation point of $\mathcal{A}_{+}$and $a \cdot$ int $(P) \subseteq$ $\operatorname{int}(P)$ for all $a \in \mathcal{A}_{+}^{*}$, if $x \in P, x \ll_{P} \varepsilon+\varepsilon$ for all $\varepsilon \in \operatorname{int}(P)$, then $x=0_{E}$;

(iv) if $\left(x_{n}\right)_{n \in \mathbb{N}},\left(y_{n}\right)_{n \in \mathbb{N}} \subset E, x, y \in E, x_{n} \stackrel{n}{\rightarrow} x, y_{n} \stackrel{n}{\rightarrow} y$, and there is a number $N \in \mathbb{N}$ such that $x_{n} \leq_{P} y_{n}$ for all $n \geq N$, then $x \leq_{P} y$.

Definition 6. Let $\left(\mathcal{A}, \oplus, \odot, \tau_{\mathcal{A}}, \preceq\right)$ be a partially ordered topological ring, $\left(E,+, \cdot, \tau_{E}\right)$ a topological left $\mathcal{A}$-module, $P$ a solid cone of $E$ and $X$ a non-empty set. A cone metric on $X$ is an application $d: X \times X \rightarrow E$ which fulfills the conditions:

(1) $0_{E} \leq_{P} d(x, y)$ for all $x, y \in X$;

(2) $d(x, y)=0_{E}$ if and only if $x=y$;

(3) $d(x, y)=d(y, x)$ for all $x, y \in X$;

(4) $d(x, y) \leq_{P} d(x, z)+d(z, y)$ for all $x, y, z \in X$.

$(X, d)$ is called a cone metric space over the topological left $\mathcal{A}$-module.

Definition 7. Let $\left(\mathcal{A}, \oplus, \odot, \tau_{\mathcal{A}}, \preceq\right)$ be a partially ordered topological ring, $\left(E,+, \cdot, \tau_{E}\right)$ a topological left $\mathcal{A}$-module, $P$ a solid cone of $E$ and $(X, d)$ a cone metric space over the topological left $\mathcal{A}$-module.

(1) A sequence $\left(x_{n}\right)_{n \in \mathbb{N}} \subset X$ is called convergent to a point $x \in X$ if it has the property: for every $\varepsilon \gg_{P} 0_{E}$ there is a number $n(\varepsilon) \in \mathbb{N}$ such that for all $n \geq n(\varepsilon)$ we have $d\left(x_{n}, x\right) \ll_{P} \varepsilon$; we also say that $\left(x_{n}\right)_{n \in \mathbb{N}} \subset X$ converges to $x \in X$ and we denote by $x_{n} \stackrel{d}{\rightarrow} x$;

(2) A sequence $\left(x_{n}\right)_{n \in \mathbb{N}} \subset X$ is named a Cauchy sequence if it satisfies the condition: for every $\varepsilon \gg_{P} 0_{E}$ there is a number $n(\varepsilon) \in \mathbb{N}$ such that for all $m, n \geq n(\varepsilon)$ we have $d\left(x_{m}, x_{n}\right) \ll_{P} \varepsilon$;

(3) The cone metric space $(X, d)$ is called complete if: any Cauchy sequence of points in $X$ is convergent in $X$.

Lemma 3. Let $\left(\mathcal{A}, \oplus, \odot, \tau_{\mathcal{A}}, \preceq\right)$ be a partially ordered Hausdorff topological ring, with $1_{\mathcal{A}} \in \mathcal{A}_{+}$, $0_{\mathcal{A}}$ an accumulation point of $\mathcal{A}_{+},\left(E,+, \cdot, \tau_{E}\right)$ a topological left $\mathcal{A}$-module, $P$ a solid cone of $E$, with $a \cdot \operatorname{int}(P) \subseteq \operatorname{int}(P)$ for all $a \in \mathcal{A}_{+}^{*}$, and $(X, d)$ a cone metric space over the topological left $\mathcal{A}$-module. If the sequence $\left(x_{n}\right)_{n \in \mathbb{N}} \subset X$ is convergent in $X$, then it has a unique limit.

Definition 8. Let $\left(E, \tau_{E}\right)$ be a topological space. A subset $S$ of $E$ is called sequentially compact if any sequence in $S$ has a convergent subsequence in $S$.

Lemma 4. Let $\left(\mathcal{A}, \oplus, \odot, \tau_{\mathcal{A}}, \preceq\right)$ be a partially ordered topological ring, with $1_{\mathcal{A}} \in \mathcal{A}_{+},\left(E,+, \cdot, \tau_{E}\right)$ a Hausdorff topological left $\mathcal{A}$-module, $P$ a solid cone of $E$ and $(X, d)$ a cone metric space over the topological left $\mathcal{A}$-module. If $\left(x_{n}\right)_{n \in \mathbb{N}} \subset X$ is not a Cauchy sequence and there is a sequentially compact subset $S_{x} \subseteq P$ having the property $\left\{d\left(x_{m}, x_{n}\right) \mid m, n \in \mathbb{N}^{*}, m<n\right\} \subseteq S_{x}$, then the following statements are valid:

(i) there exist $\varepsilon_{0} \gg_{P} 0_{E}$ and two subsequences $\left(x_{m(k)}\right)_{k \in \mathbb{N}},\left(x_{n(k)}\right)_{k \in \mathbb{N}}$, where $m, n: \mathbb{N} \rightarrow \mathbb{N}$ are strictly increasing functions, with $k<m(k)<n(k)$ for all $k \in \mathbb{N}$, such that

$$
d\left(x_{m(k)}, x_{n(k)}\right) \aleph_{P} \varepsilon_{0}, d\left(x_{m(k)}, x_{n(k)-1}\right) \ll_{P} \varepsilon_{0} \text { for all } k \in \mathbb{N} \text {; }
$$

(ii) moreover, if $\lim _{n \rightarrow+\infty} d\left(x_{n-1}, x_{n}\right)=0_{E}$, there exists a point $x \in \operatorname{Fr}(P)$ such that

$$
\lim _{k \rightarrow+\infty} d\left(x_{m(k)}, x_{n(k)}\right)=\varepsilon_{0}-x, \lim _{k \rightarrow+\infty} d\left(x_{m(k)-1}, x_{n(k)-1}\right)=\varepsilon_{0}-x \text { and } \varepsilon_{0}-x \in P .
$$


Proof. (i) Since $\left(x_{n}\right)_{n \in \mathbb{N}}$ is not a Cauchy sequence, we deduce that there is $\varepsilon_{0} \gg_{P} 0_{E}$ and two subsequences $\left(x_{m_{1}(k)}\right)_{k \in \mathbb{N}},\left(x_{\tilde{n}_{1}(k)}\right)_{k \in \mathbb{N}}$, where $m_{1}, \tilde{n}_{1}: \mathbb{N} \rightarrow \mathbb{N}$ are strictly increasing functions, with $k<m_{1}(k)<\tilde{n}_{1}(k)$ for all $k \in \mathbb{N}$, such that $d\left(x_{m_{1}(k)}, x_{\tilde{n}_{1}(k)}\right) k_{P} \varepsilon_{0}$ for all $k \in \mathbb{N}$. For every $k \in \mathbb{N}$ we choose $n_{1}(k)$ to be the smallest integer $\tilde{n}_{1}(k)$ which verifies $m_{1}(k)<n_{1}(k)$ and $d\left(x_{m_{1}(k)}, x_{n_{1}(k)}\right) \ll_{P} \varepsilon_{0}$, thus $d\left(x_{m_{1}(k)}, x_{n_{1}(k)-1}\right) \ll_{P} \varepsilon_{0}$. Hence, there exist $\varepsilon_{0} \gg_{P} 0_{E}$ and two subsequences $\left(x_{m_{1}(k)}\right)_{k \in \mathbb{N}},\left(x_{n_{1}(k)}\right)_{k \in \mathbb{N}}$, where $m_{1}, n_{1}: \mathbb{N} \rightarrow \mathbb{N}$ are strictly increasing functions, with $k<m_{1}(k)<n_{1}(k)$ for all $k \in \mathbb{N}$, such that

$$
d\left(x_{m_{1}(k)}, x_{n_{1}(k)}\right) k_{P} \varepsilon_{0}, d\left(x_{m_{1}(k)}, x_{n_{1}(k)-1}\right) \ll_{P} \varepsilon_{0} \text { for all } k \in \mathbb{N} \text {. }
$$

As $S_{x}$ is sequentially compact, any sequence in $S_{x}$ has a convergent subsequence in $S_{x}$. According to the hypothesis we have $\left\{d\left(x_{m}, x_{n}\right) \mid m, n \in \mathbb{N}^{*}, m<n\right\} \subseteq S_{x}$. Therefore, for the sequence $\left(d\left(x_{m_{1}(k)}, x_{n_{1}(k)}\right)\right)_{k \in \mathbb{N}} \subset S_{x}$ there is a subsequence $\left(d\left(x_{m_{1}(p(k))}, x_{n_{1}(p(k))}\right)\right)_{k \in \mathbb{N}}$, where $p: \mathbb{N} \rightarrow \mathbb{N}$ is a strictly increasing function, with $k<p(k)<m_{1}(p(k))<n_{1}(p(k))$ for all $k \in \mathbb{N}$, such that

$$
\lim _{k \rightarrow+\infty} d\left(x_{m_{1}(p(k))}, x_{n_{1}(p(k))}\right)=l_{1} \in S_{x} \subseteq P .
$$

Similarly, for the sequence $\left(d\left(x_{m_{1}(p(k))-1}, x_{n_{1}(p(k))-1}\right)\right)_{k \in \mathbb{N}} \subset S_{x}$ there exists a subsequence $\left(d\left(x_{m_{1}(p(q(k)))-1}, x_{n_{1}(p(q(k)))-1}\right)\right)_{k \in \mathbb{N}}$, where $q: \mathbb{N} \rightarrow \mathbb{N}$ is a strictly increasing function, with $k<q(k)<p(q(k))<m_{1}(p(q(k)))<n_{1}(p(q(k)))$ for all $k \in \mathbb{N}$, such that

$$
\lim _{k \rightarrow+\infty} d\left(x_{m_{1}(p(q(k)))-1}, x_{n_{1}(p(q(k)))-1}\right)=l_{2} \in S_{x} \subseteq P .
$$

Applying the statement (1) for $k:=p(q(k)), k \in \mathbb{N}$, it follows that there exist $\varepsilon_{0} \gg_{P} 0_{E}$ and two subsequences $\left(x_{m(k)}\right)_{k \in \mathbb{N}},\left(x_{n(k)}\right)_{k \in \mathbb{N}}$, where $m, n: \mathbb{N} \rightarrow \mathbb{N}, m(k)=m_{1}(p(q(k)))$, $n(k)=n_{1}(p(q(k)))$ are strictly increasing functions, with $k<m(k)<n(k)$ for all $k \in \mathbb{N}$, such that $d\left(x_{m(k)}, x_{n(k)}\right) \ll_{P} \varepsilon_{0}, d\left(x_{m(k)}, x_{n(k)-1}\right) \ll_{P} \varepsilon_{0}$ for all $k \in \mathbb{N}$.

(ii) As $\tau_{E}$ is a Hausdorff topology, every convergent sequence in $E$ has a unique limit in $E$.

Considering (i) we get $d\left(x_{m(k)}, x_{n(k)}\right) k_{P} \varepsilon_{0}$ for all $k \in \mathbb{N}$, hence $\varepsilon_{0}-d\left(x_{m(k)}, x_{n(k)}\right) \in$ $c(\operatorname{int}(P))$ for all $k \in \mathbb{N}$. Since $\left(d\left(x_{m(k)}, x_{n(k)}\right)\right)_{k \in \mathbb{N}}$ represents the sequence $\left(d\left(x_{m_{1}}(p(q(k)))\right.\right.$, $\left.\left.x_{n_{1}(p(q(k)))}\right)\right)_{k \in \mathbb{N}}$ which is a subsequence of $\left(d\left(x_{m_{1}(p(k))}, x_{n_{1}(p(k))}\right)\right)_{k \in \mathbb{N}}$ and taking into account that $\lim _{k \rightarrow+\infty} d\left(x_{m_{1}(p(k))}, x_{n_{1}(p(k))}\right)=l_{1}$ (according to the relation (2)), we deduce $\lim _{k \rightarrow+\infty} d\left(x_{m(k)}, x_{n(k)}\right)=l_{1}$. We find $\lim _{k \rightarrow+\infty}\left(\varepsilon_{0}-d\left(x_{m(k)}, x_{n(k)}\right)\right)=\varepsilon_{0}-l_{1}$. Therefore, $\varepsilon_{0}-l_{1} \in$ $\frac{k \rightarrow+\infty}{c(\operatorname{int}(P))}=c(\operatorname{int}(P))(\operatorname{int}(P)$ is an open set, hence $c(\operatorname{int}(P))$ is a closed set). Taking into account the properties of the cone metric $d$, the statement (i) and using Lemma 1 (iii) we obtain

$$
\begin{gathered}
d\left(x_{m(k)}, x_{n(k)}\right) \leq_{P} d\left(x_{m(k)}, x_{n(k)-1}\right)+d\left(x_{n(k)-1}, x_{n(k)}\right) \ll_{P} \\
\varepsilon_{0}+d\left(x_{n(k)-1}, x_{n(k)}\right) \text { for all } k \in \mathbb{N},
\end{gathered}
$$

thus

$$
\varepsilon_{0}+d\left(x_{n(k)-1}, x_{n(k)}\right)-d\left(x_{m(k)}, x_{n(k)}\right) \in \operatorname{int}(P) \text { for all } k \in \mathbb{N} .
$$

As $\left(d\left(x_{n(k)-1}, x_{n(k)}\right)\right)_{k \in \mathbb{N}}$ is a subsequence of $\left(d\left(x_{n-1}, x_{n}\right)\right)_{n \in \mathbb{N}}$ and $\lim _{n \rightarrow+\infty} d\left(x_{n-1}, x_{n}\right)=$ $0_{E}$ we deduce $\lim _{k \rightarrow+\infty} d\left(x_{n(k)-1}, x_{n(k)}\right)=0_{E}$; also, $\lim _{k \rightarrow+\infty} d\left(x_{m(k)}, x_{n(k)}\right)=l_{1}$. Therefore, $\lim _{k \rightarrow+\infty}\left(\varepsilon_{0}+d\left(x_{n(k)-1}, x_{n(k)}\right)-d\left(x_{m(k)}, x_{n(k)}\right)\right)=\varepsilon_{0}-l_{1}$. It follows that $\varepsilon_{0}-l_{1} \in \overline{\text { int }(P)} \subseteq$ $\bar{P}=P$, hence $\varepsilon_{0}-l_{1} \in c(\operatorname{int}(P)) \cap P=P \cap c(\operatorname{int}(P))=\bar{P} \cap c(\operatorname{int}(P))=F r(P)$. Consequently, there exists a point $x \in \operatorname{Fr}(P)$ such that $\varepsilon_{0}-l_{1}=x$, thus $l_{1}=\varepsilon_{0}-x$, so $\lim _{k \rightarrow+\infty} d\left(x_{m(k)}, x_{n(k)}\right)=\varepsilon_{0}-x$.

The properties of the cone metric $d$ leads to

$$
d\left(x_{m(k)-1}, x_{n(k)-1}\right) \leq_{P} d\left(x_{m(k)-1}, x_{m(k)}\right)+d\left(x_{m(k)}, x_{n(k)}\right)+d\left(x_{n(k)}, x_{n(k)-1}\right) \text { for all } k \in \mathbb{N}
$$


and

$$
d\left(x_{m(k)}, x_{n(k)}\right) \leq_{P} d\left(x_{m(k)}, x_{m(k)-1}\right)+d\left(x_{m(k)-1}, x_{n(k)-1}\right)+d\left(x_{n(k)-1}, x_{n(k)}\right) \text { for all } k \in \mathbb{N} .
$$

Since $\left(d\left(x_{m(k)-1}, x_{n(k)-1}\right)\right)_{k \in \mathbb{N}}$ represents the sequence $\left(d\left(x_{m_{1}(p(q(k)))-1}\right.\right.$, $\left.\left.x_{n_{1}(p(q(k)))-1}\right)\right)_{k \in \mathbb{N}}$ and $\lim _{k \rightarrow+\infty} d\left(x_{m_{1}(p(q(k)))-1}, x_{n_{1}(p(q(k)))-1}\right)=l_{2}$ (according to the relation (3)), we find $\lim _{k \rightarrow+\infty} d\left(x_{m(k)-1}, x_{n(k)-1}\right)=l_{2}$. Also, $\left(d\left(x_{m(k)-1}, x_{m(k)}\right)\right)_{k \in \mathbb{N}},\left(d\left(x_{n(k)-1}\right.\right.$, $\left.\left.x_{n(k)}\right)\right)_{k \in \mathbb{N}}$ are subsequences of $\left(d\left(x_{n-1}, x_{n}\right)\right)_{n \in \mathbb{N}}$ and $\lim _{n \rightarrow+\infty} d\left(x_{n-1}, x_{n}\right)=0_{E}$, hence $\lim _{k \rightarrow+\infty} d\left(x_{m(k)-1}, x_{m(k)}\right)=\lim _{k \rightarrow+\infty} d\left(x_{n(k)-1}, x_{n(k)}\right)=0_{E}$; moreover, $\lim _{k \rightarrow+\infty} d\left(x_{m(k)}, x_{n(k)}\right)=$ $\varepsilon_{0}-x$. Passing to the limit for the sequences in relations (4), (5), we obtain $l_{2} \leq_{P} \varepsilon_{0}-x$ and $\varepsilon_{0}-x \leq_{P} l_{2}$, thus $l_{2}=\varepsilon_{0}-x$, so $\lim _{k \rightarrow+\infty} d\left(x_{m(k)-1}, x_{n(k)-1}\right)=\varepsilon_{0}-x$.

As $\left(d\left(x_{m(k)}, x_{n(k)}\right)\right)_{k \in \mathbb{N}} \subset P$ and $\lim _{k \rightarrow+\infty} d\left(x_{m(k)}, x_{n(k)}\right)=\varepsilon_{0}-x$, it follows that $\varepsilon_{0}-x \in$ $\bar{P}=P(P$ being a closed set $)$.

Consequently, we proved that there exists a point $x \in \operatorname{Fr}(P)$ such that $\lim _{k \rightarrow+\infty} d\left(x_{m(k)}, x_{n(k)}\right)=\varepsilon_{0}-x, \lim _{k \rightarrow+\infty} d\left(x_{m(k)-1}, x_{n(k)-1}\right)=\varepsilon_{0}-x$ and $\varepsilon_{0}-x \in P$.

In the following, we define the operation

$$
*: \mathbb{N} \times E \rightarrow E, n * t= \begin{cases}0_{E}, & n=0 \\ \underbrace{t+\cdots+t}_{n}, & n \geq 1 .\end{cases}
$$

Definition 9. Let $\left(\mathcal{A}, \oplus, \odot, \tau_{\mathcal{A}}, \preceq\right)$ be a partially ordered topological ring, $\left(E,+, \cdot, \tau_{E}\right)$ a topological left $\mathcal{A}$-module and $P$ a solid cone of $E$. A sequence $\left(u_{n}\right)_{n \in \mathbb{N}} \subset E$ is called:

(1) an increasing sequence if $u_{n-1} \leq_{P} u_{n}$ for all $n \in \mathbb{N}^{*}$;

(2) a decreasing sequence if $u_{n-1} \geq_{P} u_{n}$ for all $n \in \mathbb{N}^{*}$.

Definition 10. Let $\left(E, \tau_{E}\right)$ be a topological space, $S$ a subset of $E$ and $F: S \rightarrow E$ a function.

(1) $F$ is sequentially continuous at a point $x \in S$ if: for every sequence $\left(x_{n}\right)_{n \in \mathbb{N}} \subset S$ convergent to $x$, the sequence $\left(F\left(x_{n}\right)\right)_{n \in \mathbb{N}} \subset E$ is convergent to $F(x)$;

(2) $F$ is named sequentially continuous on $S$ if: it is sequentially continuous at every point $x \in S$.

Definition 11. Let $\left(\mathcal{A}, \oplus, \odot, \tau_{\mathcal{A}}, \preceq\right)$ be a partially ordered topological ring, $\left(E,+, \cdot, \tau_{E}\right)$ a topological left $\mathcal{A}$-module and $P$ a solid cone of $E$. We consider the set $\mathcal{F}^{\prime}$ of all functions $F: P^{*} \rightarrow E$ which fulfill the conditions:

(1) if $u \in P^{*}, v \in \operatorname{int}(P)$, then for every $\varepsilon \gg_{P} 0_{E}$ there is a number $n(\varepsilon, u, v) \in \mathbb{N}$ such that for all $n \geq n(\varepsilon, u, v)$ we have $F(u)-n * v \ll_{P}-\varepsilon$;

(2) if $\left(u_{n}\right)_{n \in \mathbb{N}} \subset P^{*}$ is a decreasing sequence and for every $\varepsilon \gg_{P} 0_{E}$ there is a number $n(\varepsilon) \in \mathbb{N}$ such that for all $n \geq n(\varepsilon)$ we have $F\left(u_{n}\right) \ll_{P}-\varepsilon$, then $u_{n} \stackrel{n}{\rightarrow} 0_{E}$;

(3) if $u, v \in P^{*}, F(u) \ll_{P} F(v)$, then $u \leq_{P} v$;

(4) $F$ is sequentially continuous on $P^{*}$.

Definition 12. Let $\left(\mathcal{A}, \oplus, \odot, \tau_{\mathcal{A}}, \preceq\right)$ be a partially ordered topological ring, $\left(E,+, \cdot, \tau_{E}\right)$ a topological left $\mathcal{A}$-module, $P$ a solid cone of $E$ and $(X, d)$ a cone metric space over the topological left $\mathcal{A}$-module. An F-contraction corresponding to the function class $\mathcal{F}^{\prime}$ is a mapping $T: X \rightarrow X$ for which there exist $\tau \gg_{P} 0_{E}$ and a function $F \in \mathcal{F}^{\prime}$ such that

$$
\tau+F(d(T x, T y)) \leq{ }_{P} F(d(x, y)), \text { for all } x, y \in X, T x \neq T y .
$$

Remark 1. The condition $T x \neq T y$ from the property (6) implies $x \neq y$. Hence, $d(x, y) \in P^{*}$ whenever $d(T x, T y) \in P^{*}$. Therefore, the function $F \in \mathcal{F}^{\prime}$ is defined for every $x, y \in X$ satisfying the condition $T x \neq T y$. 
Theorem 1. Let $\left(\mathcal{A}, \oplus, \odot, \tau_{\mathcal{A}}, \preceq\right)$ be a partially ordered Hausdorff topological ring, with $1_{\mathcal{A}} \in \mathcal{A}_{+}$, $0_{\mathcal{A}}$ an accumulation point of $\mathcal{A}_{+},\left(E,+, \cdot, \tau_{E}\right)$ a Hausdorff topological left $\mathcal{A}$-module, $P$ a solid cone of $E$, with $a \cdot \operatorname{int}(P) \subseteq \operatorname{int}(P)$ for all $a \in \mathcal{A}_{+}^{*}, 0_{E} \notin \operatorname{int}(P)$, and $(X, d)$ a cone metric space over the topological left $\mathcal{A}$-module. We suppose that $(X, d)$ is complete, $T: X \rightarrow X$ is an $F$-contraction corresponding to the function class $\mathcal{F}^{\prime}$ and for every $x_{0} \in X$ we consider the sequence $\left(x_{n}\right)_{n \in \mathbb{N}} \subset X$ defined by $x_{n}=T x_{n-1}$ for all $n \in \mathbb{N}^{*}$. If for any $x_{0} \in X$ for which $\left(x_{n}\right)_{n \in \mathbb{N}} \subset X$ is not a Cauchy sequence, there is a sequentially compact subset $S_{x_{0}} \subseteq P$ having the property $\left\{d\left(x_{m}, x_{n}\right) \mid m, n \in \mathbb{N}^{*}, m<n\right\} \subseteq S_{x_{0}}$, then $T$ has a unique fixed point $x^{\star} \in X$ and for every $x_{0} \in X$ the sequence $\left(x_{n}\right)_{n \in \mathbb{N}} \subset X$ converges to $x^{\star}$.

Proof. Let us suppose that $T$ has two distinct fixed points, so there exist $x_{1}, x_{2} \in X$ such that $T x_{1}=x_{1} \neq x_{2}=T x_{2}$. Because $T: X \rightarrow X$ is an $F$-contraction corresponding to the function class $\mathcal{F}^{\prime}$ we deduce that there exist $\tau \gg_{P} 0_{E}$ and a function $F \in \mathcal{F}^{\prime}$ such that the property (6) is verified. Applying this property we get $\tau+F\left(d\left(T x_{1}, T x_{2}\right)\right) \leq_{P} F\left(d\left(x_{1}, x_{2}\right)\right)$, thus $\left.\tau+F\left(d\left(x_{1}, x_{2}\right)\right) \leq_{P} F\left(d\left(x_{1}, x_{2}\right)\right)\right)$, so $\tau \leq_{P} 0_{E}$, hence $\tau \in(-P)$. It follows that $\tau \in \operatorname{int}(P) \cap(-P)$. On the other hand, int $(P) \cap(-P)=\varnothing$ (according to Lemma 2 (i)), consequently $\tau \in \varnothing$, which is in contradiction with $\tau \gg_{P} 0_{E}$. Therefore, $T$ has at most one fixed point.

We choose $x_{0} \in X$ be an arbitrary element and let us define the sequence $\left(x_{n}\right)_{n \in \mathbb{N}} \subset X$ by the recurrence relation $x_{n}=T x_{n-1}$ for all $n \in \mathbb{N}^{*}$. To prove the existence of a fixed point of the operator $\mathrm{T}$ we distinguish the following cases:

I: there is $N \in \mathbb{N}^{*}$ such that $x_{N}=x_{N-1}$. We deduce $T x_{N-1}=x_{N-1}$, thus $x_{N-1}$ is a fixed point of $T$. Considering that $T$ has at most one fixed point, we get $x^{\star}:=x_{N-1} \in X$ is the unique fixed point of $T$. Moreover, $x_{n}=x_{N-1}$ for all $n \geq N$, which means that the sequence $\left(x_{n}\right)_{n \in \mathbb{N}} \subset X$ converges to $x^{\star}$.

II: $x_{n} \neq x_{n-1}$ for all $n \in \mathbb{N}^{*}$. Let us consider $n \in \mathbb{N}^{*}$ be an arbitrary element. Using the property (6) we find $\tau+F\left(d\left(T x_{k-1}, T x_{k}\right)\right) \leq_{P} F\left(d\left(x_{k-1}, x_{k}\right)\right), k=\overline{1, n}$, thus $\tau+F\left(d\left(x_{k}, x_{k+1}\right)\right) \leq_{P} F\left(d\left(x_{k-1}, x_{k}\right)\right), k=\overline{1, n}$. Following Lemma 1 (iv) we deduce $n *$ $\tau+\sum_{k=1}^{n} F\left(d\left(x_{k}, x_{k+1}\right)\right) \leq_{P} \sum_{k=1}^{n} F\left(d\left(x_{k-1}, x_{k}\right)\right)$, thus $n * \tau+F\left(d\left(x_{n}, x_{n+1}\right)\right) \leq_{P} F\left(d\left(x_{0}, x_{1}\right)\right)$. Therefore, we get

$$
F\left(d\left(x_{n}, x_{n+1}\right)\right) \leq_{P} F\left(d\left(x_{0}, x_{1}\right)\right)-n * \tau \text { for all } n \in \mathbb{N}^{*} .
$$

On the other hand, $F \in \mathcal{F}^{\prime}$, hence from Definition 11 (1) we obtain for every $\varepsilon \gg_{P} 0_{E}$ there is a number $n\left(\varepsilon, d\left(x_{0}, x_{1}\right), \tau\right) \in \mathbb{N}$ such that for all $n \geq n\left(\varepsilon, d\left(x_{0}, x_{1}\right), \tau\right)$ we have $F\left(d\left(x_{0}, x_{1}\right)\right)-n * \tau \ll_{P}-\varepsilon$. Consequently, from the previous affirmation and using the property (7), via Lemma 1 (iii) we find:

for every $\varepsilon \gg_{P} 0_{E}$ there is a number $n\left(\varepsilon, d\left(x_{0}, x_{1}\right), \tau\right) \in \mathbb{N}$ such that for all $n \geq$ $n\left(\varepsilon, d\left(x_{0}, x_{1}\right), \tau\right)$ we have

$$
F\left(d\left(x_{n}, x_{n+1}\right)\right) \ll_{P}-\varepsilon .
$$

Let us consider $n \in \mathbb{N}^{*}$ be an arbitrary number. Using the property (6) we get $\tau+F\left(d\left(T x_{n-1}, T x_{n}\right)\right) \leq_{P} F\left(d\left(x_{n-1}, x_{n}\right)\right)$, so $F\left(d\left(x_{n}, x_{n+1}\right)\right) \leq_{P} F\left(d\left(x_{n-1}, x_{n}\right)\right)-\tau$. Since $\tau \gg_{P} 0_{E}$ it follows that $F\left(d\left(x_{n}, x_{n+1}\right)\right) \ll_{P} F\left(d\left(x_{n-1}, x_{n}\right)\right)$. As $F \in \mathcal{F}^{\prime}$, from Definition 11 (3) we obtain $d\left(x_{n}, x_{n+1}\right) \leq_{P} d\left(x_{n-1}, x_{n}\right)$, thus $\left(d\left(x_{n}, x_{n+1}\right)\right)_{n \in \mathbb{N}}$ is a decreasing sequence. Considering Definition 11 (2) and the property (8), we deduce $d\left(x_{n}, x_{n+1}\right) \stackrel{n}{\rightarrow} 0_{E}$, so $d\left(x_{n-1}, x_{n}\right) \stackrel{n}{\rightarrow} 0_{E}$. In the following, we prove that $\left(x_{n}\right)_{n \in \mathbb{N}} \subset X$ is a Cauchy sequence. Let us suppose that $\left(x_{n}\right)_{n \in \mathbb{N}} \subset X$ is not a Cauchy sequence. Since for $x_{0} \in X$ for which $\left(x_{n}\right)_{n \in \mathbb{N}} \subset X$ is not a Cauchy sequence, there is a sequentially compact subset $S_{x_{0}} \subseteq P$ having the property $\left\{d\left(x_{m}, x_{n}\right) \mid m, n \in \mathbb{N}^{*}, m<n\right\} \subseteq S_{x_{0}}$ and considering $d\left(x_{n-1}, x_{n}\right) \stackrel{n}{\rightarrow} 0_{E}$, using Lemma 4 (i), (ii) we deduce there exist $\varepsilon_{0} \gg_{P} 0_{E}$, two subsequences $\left(x_{m(k)}\right)_{k \in \mathbb{N}}$, 
$\left(x_{n(k)}\right)_{k \in \mathbb{N}}$, where $m, n: \mathbb{N} \rightarrow \mathbb{N}$ are strictly increasing functions, with $k<m(k)<n(k)$ for all $k \in \mathbb{N}$, and a point $x \in \operatorname{Fr}(P)$ such that

$$
\lim _{k \rightarrow+\infty} d\left(x_{m(k)}, x_{n(k)}\right)=\varepsilon_{0}-x, \lim _{k \rightarrow+\infty} d\left(x_{m(k)-1}, x_{n(k)-1}\right)=\varepsilon_{0}-x \text { and } \varepsilon_{0}-x \in P .
$$

As $\varepsilon_{0} \in \operatorname{int}(P), x \in \operatorname{Fr}(P)$ and according to Lemma 2 (ii), $\operatorname{int}(P) \cap \operatorname{Fr}(P)=\varnothing$, we get $\varepsilon_{0}-x \neq 0_{E}$. Considering relation (9), it follows that there is a number $K \in \mathbb{N}$ such that $d\left(x_{m(k)}, x_{n(k)}\right) \neq 0_{E}$ for all $k \geq K$, hence $x_{m(k)} \neq x_{n(k)}$ for all $k \geq K$. Using the property (6) we find $\tau+F\left(d\left(T x_{m(k)-1}, T x_{n(k)-1}\right)\right) \leq_{P} F\left(d\left(x_{m(k)-1}, x_{n(k)-1}\right)\right)$ for all $k \geq K$, hence

$$
\tau+F\left(d\left(x_{m(k)}, x_{n(k)}\right)\right) \leq_{P} F\left(d\left(x_{m(k)-1}, x_{n(k)-1}\right)\right) \text { for all } k \geq K
$$

On the other hand, as $F \in \mathcal{F}^{\prime}$, from Definition 11 (4) we have $F$ is sequentially continuous on $P^{*}$, hence $F$ is sequentially continuous at $\varepsilon_{0}-x \in P^{*}$. Considering the property (9) we obtain

$$
\lim _{k \rightarrow+\infty} F\left(d\left(x_{m(k)}, x_{n(k)}\right)\right)=F\left(\varepsilon_{0}-x\right), \lim _{k \rightarrow+\infty} F\left(d\left(x_{m(k)-1}, x_{n(k)-1}\right)\right)=F\left(\varepsilon_{0}-x\right) .
$$

Passing to the limit in inequality (10) and considering the relation (11), we deduce $\tau+F\left(\varepsilon_{0}-x\right) \leq_{P} F\left(\varepsilon_{0}-x\right)$, thus $\tau \leq_{P} 0_{E}$, so $\tau \in(-P)$. It follows that $\tau \in \operatorname{int}(P) \cap(-P)$. On the other hand, $\operatorname{int}(P) \cap(-P)=\varnothing$ (according to Lemma 2 (i)), therefore $\tau \in \varnothing$ which is in contradiction with $\tau \gg_{P} 0_{E}$. Consequently, $\left(x_{n}\right)_{n \in \mathbb{N}} \subset X$ is a Cauchy sequence. Since $(X, d)$ is a complete cone metric space, we deduce that there exists an element $x^{\star} \in X$ such that the sequence $\left(x_{n}\right)_{n \in \mathbb{N}} \subset X$ converges to $x^{\star} \in X$.

Further, we show that $x^{\star}$ is a fixed point of $T$. For this, we consider the set $U=\{n \in$ $\left.\mathbb{N} \mid x_{n}=T x^{\star}\right\}$ and we distinguish the following subcases:

II.1: $U$ is an infinite set. We can choose a subsequence $\left(x_{m(k)}\right)_{k \in \mathbb{N}}$ of $\left(x_{n}\right)_{n \in \mathbb{N}}$, where $m: \mathbb{N} \rightarrow \mathbb{N}$ is a strictly increasing function, with $k<m(k)$ for all $k \in \mathbb{N}$, such that $\left(x_{m(k)}\right)_{k \in \mathbb{N}}$ converges to $T x^{\star}$. However, we show that the sequence $\left(x_{n}\right)_{n \in \mathbb{N}}$ converges to $x^{\star}$. Considering Lemma 3 we obtain that the sequence $\left(x_{n}\right)_{n \in \mathbb{N}}$ has a unique limit, hence $T x^{\star}=x^{\star}$, so $x^{\star}$ is a fixed point of $T$.

II.2: $H$ is a finite set. We find that there exists a number $N \in \mathbb{N}$ such that $x_{n} \neq T x^{\star}$ for all $n \geq N$. Let us consider $n \geq N$ be an arbitrary element. Using the property (6) we obtain $\tau+F\left(d\left(T x_{n-1}, T x^{\star}\right)\right) \leq_{P} F\left(d\left(x_{n-1}, x^{\star}\right)\right)$, thus $F\left(d\left(x_{n}, T x^{\star}\right)\right) \leq_{P} F\left(d\left(x_{n-1}, x^{\star}\right)\right)-\tau$. Since $\tau \gg_{P} 0_{E}$ it follows that $F\left(d\left(x_{n}, T x^{\star}\right)\right) \ll_{P} F\left(d\left(x_{n-1}, x^{\star}\right)\right)$. As $F \in \mathcal{F}^{\prime}$, from Definition 11 (3) we obtain

$$
d\left(x_{n}, T x^{\star}\right) \leq_{P} d\left(x_{n-1}, x^{\star}\right) \text { for all } n \geq N .
$$

Further, the properties of the cone metric $d$ lead to

$$
d\left(x^{\star}, T x^{\star}\right) \leq_{P} d\left(x^{\star}, x_{n}\right)+d\left(x_{n}, T x^{\star}\right)=d\left(x_{n}, x^{\star}\right)+d\left(x_{n}, T x^{\star}\right) \text { for all } n \in \mathbb{N} \text {. }
$$

Taking into account the relations (12), (13) we get

$$
d\left(x^{\star}, T x^{\star}\right) \leq_{P} d\left(x_{n}, x^{\star}\right)+d\left(x_{n-1}, x^{\star}\right) \text { for all } n \geq N \text {. }
$$

We choose $\varepsilon \in \operatorname{int}(P)$ be an arbitrary element. Since $\left(x_{n}\right)_{n \in \mathbb{N}} \subset X$ converges to $x^{\star} \in X$, we deduce that there is a number $n(\varepsilon) \in \mathbb{N}$ such that for all $n \geq n(\varepsilon)$ we have $d\left(x_{n}, x^{\star}\right) \ll_{P} \varepsilon$. It follows that

$$
d\left(x_{n}, x^{\star}\right)+d\left(x_{n-1}, x^{\star}\right) \ll_{P} \varepsilon+\varepsilon \text { for all } n \geq n(\varepsilon)+1
$$

Let us consider $n \geq \max \{n(\varepsilon)+1, N\}$ to be a natural number. Using the relations (14), (15) and taking into account Lemma 1 (iii) we obtain

$$
d\left(x^{\star}, T x^{\star}\right) \ll_{P} \varepsilon+\varepsilon .
$$


Therefore, $d\left(x^{\star}, T x^{\star}\right) \in P$ and $d\left(x^{\star}, T x^{\star}\right) \ll_{P} \varepsilon+\varepsilon$ for all $\varepsilon \in \operatorname{int}(P)$. Applying Lemma 2 (iii) it follows that $d\left(x^{\star}, T x^{\star}\right)=0_{E}$, thus $x^{\star}=T x^{\star}$, hence $x^{\star}$ is a fixed point of $T$.

Consequently, in both subcases II.1 and II.2 we showed that $x^{\star} \in X$ is a fixed point of $T$. Considering that $T$ has at most one fixed point, we obtain that $x^{\star} \in X$ is the unique fixed point of $T$. Also, we proved that $\left(x_{n}\right)_{n \in \mathbb{N}}$ converges to $x^{\star}$.

Corollary 1. Let $\left(\mathcal{A}, \oplus, \odot, \tau_{\mathcal{A}}, \preceq\right)$ be a partially ordered Hausdorff topological ring, with $1_{\mathcal{A}} \in$ $\mathcal{A}_{+}, 0_{\mathcal{A}}$ an accumulation point of $\mathcal{A}_{+},\left(E,+, \cdot, \tau_{E}\right)$ a Hausdorff topological left $\mathcal{A}$-module, $P$ a solid cone of $E$, with $a \cdot \operatorname{int}(P) \subseteq \operatorname{int}(P)$ for all $a \in \mathcal{A}_{+}^{*}, 0_{E} \notin \operatorname{int}(P)$, and $(X, d)$ a cone metric space over the topological left $\mathcal{A}$-module. We suppose that $(X, d)$ is complete, $T: X \rightarrow X$ is an $F$-contraction corresponding to the function class $\mathcal{F}^{\prime}$ and for every $x_{0} \in X$ we consider the sequence $\left(x_{n}\right)_{n \in \mathbb{N}} \subset X$ defined by $x_{n}=T x_{n-1}$ for all $n \in \mathbb{N}^{*}$. If there is a sequentially compact subset $S \subseteq P$ having the property $\{d(T x, T y) \mid x, y \in X\} \subseteq S$, then $T$ has a unique fixed point $x^{\star} \in X$ and for every $x_{0} \in X$ the sequence $\left(x_{n}\right)_{n \in \mathbb{N}} \subset X$ converges to $x^{\star}$.

Proof. Let $x_{0} \in X$ for which $\left(x_{n}\right)_{n \in \mathbb{N}} \subset X$ is not a Cauchy sequence, be an arbitrary element. Because for every $m, n \in \mathbb{N}^{*}, m<n$, we have $x_{m}=T x_{m-1}, x_{n}=T x_{n-1}$, it follows that $\left\{d\left(x_{m}, x_{n}\right) \mid m, n \in \mathbb{N}^{*}, m<n\right\} \subseteq\{d(T x, T y) \mid x, y \in X\}$. According to the hypothesis, there is a sequentially compact subset $S \subseteq P$ having the property $\{d(T x, T y) \mid x, y \in X\} \subseteq S$, hence $\left\{d\left(x_{m}, x_{n}\right) \mid m, n \in \mathbb{N}^{*}, m<n\right\} \subseteq S$. Therefore, for any $x_{0} \in X$ for which $\left(x_{n}\right)_{n \in \mathbb{N}} \subset X$ is not a Cauchy sequence, there is a sequentially compact subset $S_{x_{0}}:=S \subseteq P$ having the property $\left\{d\left(x_{m}, x_{n}\right) \mid m, n \in \mathbb{N}^{*}, m<n\right\} \subseteq S_{x_{0}}$. Consequently, the hypotheses of Theorem 1 are fulfilled, hence $T$ has a unique fixed point $x^{\star} \in X$ and for every $x_{0} \in X$ the sequence $\left(x_{n}\right)_{n \in \mathbb{N}} \subset X$ converges to $x^{\star}$.

In the following, we determine some conditions for the existence and uniqueness of a solution of the following integral equation

$$
x(t)=\int_{a}^{t} K(t, s, x(s)) d s+f(t), t \in[a, b],
$$

where $x:[a, b] \rightarrow \mathbb{R}^{n}, K:[a, b] \times[a, b] \times \mathbb{R}^{n} \rightarrow \mathbb{R}^{n}, f:[a, b] \rightarrow \mathbb{R}^{n}$, by using Corollary 1 .

Theorem 2. If the functions $K:[a, b] \times[a, b] \times \mathbb{R}^{n} \rightarrow \mathbb{R}^{n}, f:[a, b] \rightarrow \mathbb{R}^{n}$ are continuous and there is $\tau>0$ such that

$$
\left|K_{i}(t, s, x(s))-K_{i}(t, s, y(s))\right| \leq \frac{1}{b-a} \frac{\left|x_{i}(t)-y_{i}(t)\right|}{\tau d(x, y)+1},
$$

for all $t, s \in[a, b], x, y \in C\left([a, b], \mathbb{R}^{n}\right), i=\overline{1, n}$, then the integral Equation (16) has a unique solution in $C\left([a, b], \mathbb{R}^{n}\right)$.

Proof. Let $\left(\mathbb{R},+, \cdot, \tau_{\mathbb{R}}, \leq\right)$ be the partially ordered Hausdorff topological ring and $\left(\mathbb{R},+, \cdot, \tau_{\mathbb{R}}\right)$ the Hausdorff topological left $\mathbb{R}$-module, where $\tau_{\mathbb{R}}$ is the Euclidean topology. We consider the solid cone $P=\mathbb{R}_{+}$, with $\operatorname{int}(P)=\mathbb{R}_{+}^{*}$. We define the set $C\left([a, b], \mathbb{R}^{n}\right)=$ $\left\{x:[a, b] \rightarrow \mathbb{R}^{n} \mid x\right.$ is continuous on $\left.[a, b]\right\}$ and the mapping

$$
d: C\left([a, b], \mathbb{R}^{n}\right) \times C\left([a, b], \mathbb{R}^{n}\right) \rightarrow \mathbb{R}_{+}, d(x, y)=\|x-y\|,
$$

where $\|\cdot\|: C\left([a, b], \mathbb{R}^{n}\right) \rightarrow \mathbb{R}_{+},\|x\|=\max _{i=1, n} \max _{t \in[a, b]}\left(\left|x_{i}(t)\right| e^{-\tau(t-a)}\right)$. We remark that $\left(C\left([a, b], \mathbb{R}^{n}\right), d\right)$ is a complete cone metric space over the topological left $\mathbb{R}$-module. Next, we consider the function

$$
F: \mathbb{R}_{+}^{*} \rightarrow \mathbb{R}, F(x)=-\frac{1}{x}
$$

which belongs to the class $\mathcal{F}^{\prime}$. 
Since the functions $K:[a, b] \times[a, b] \times \mathbb{R}^{n} \rightarrow \mathbb{R}^{n}$ and $f:[a, b] \rightarrow \mathbb{R}^{n}$ are continuous, we can define the operator $T: C\left([a, b], \mathbb{R}^{n}\right) \rightarrow C\left([a, b], \mathbb{R}^{n}\right)$,

$$
T(x)(t)=\int_{a}^{t} K(t, s, x(s)) d s+f(t), t \in[a, b] .
$$

A function $x \in C\left([a, b], \mathbb{R}^{n}\right)$ is a solution of the integral Equation (16) if and only if it is a fixed point of the operator $T$.

Let $t, s \in[a, b], x, y \in C\left([a, b], \mathbb{R}^{n}\right), i=\overline{1, n}$, be arbitrary elements.

The definition of the operator $T$ leads to

$$
\begin{gathered}
\left|T_{i}(x)(t)-T_{i}(y)(t)\right| \\
=\left|\int_{a}^{t} K_{i}(t, s, x(s)) d s+f_{i}(t)-\int_{a}^{t} K_{i}(t, s, y(s)) d s-f_{i}(t)\right| \\
=\left|\int_{a}^{t}\left(K_{i}(t, s, x(s))-K_{i}(t, s, y(s))\right) d s\right| \leq \int_{a}^{t}\left|K_{i}(t, s, x(s))-K_{i}(t, s, y(s))\right| d s .
\end{gathered}
$$

Considering the hypothesis we find

$$
\begin{gathered}
\left|K_{i}(t, s, x(s))-K_{i}(t, s, y(s))\right| \leq \frac{1}{b-a} \frac{\left|x_{i}(t)-y_{i}(t)\right|}{\tau d(x, y)+1} \\
=\frac{1}{b-a} \frac{\left|x_{i}(t)-y_{i}(t)\right| e^{-\tau(t-a)} e^{\tau(t-a)}}{\tau d(x, y)+1} \leq \frac{1}{b-a} \frac{\max _{t \in[a, b]}\left(\left|x_{i}(t)-y_{i}(t)\right| e^{-\tau(t-a)}\right) e^{\tau(t-a)}}{\tau d(x, y)+1} \\
\leq \frac{1}{b-a} \frac{\max _{i=1, n} \max _{t \in[a, b]}\left(\left|x_{i}(t)-y_{i}(t)\right| e^{-\tau(t-a)}\right) e^{\tau(t-a)}}{\tau d(x, y)+1} \\
=\frac{1}{b-a} \frac{\|x-y\| e^{\tau(t-a)}}{\tau d(x, y)+1}=\frac{1}{b-a} \frac{d(x, y) e^{\tau(t-a)}}{\tau d(x, y)+1},
\end{gathered}
$$

thus

$$
\begin{gathered}
\int_{a}^{t}\left|K_{i}(t, s, x(s))-K_{i}(t, s, y(s))\right| d s \leq \int_{a}^{t} \frac{1}{b-a} \frac{d(x, y) e^{\tau(t-a)}}{\tau d(x, y)+1} d s \\
=\frac{1}{b-a} \frac{d(x, y) e^{\tau(t-a)}}{\tau d(x, y)+1} \int_{a}^{t} d s=\left.\frac{1}{b-a} \frac{d(x, y) e^{\tau(t-a)}}{\tau d(x, y)+1} s\right|_{a} ^{t} \\
=\frac{1}{b-a} \frac{d(x, y) e^{\tau(t-a)}}{\tau d(x, y)+1}(t-a) \leq \frac{1}{b-a} \frac{d(x, y) e^{\tau(t-a)}}{\tau d(x, y)+1}(b-a)=\frac{d(x, y) e^{\tau(t-a)}}{\tau d(x, y)+1} .
\end{gathered}
$$

From the previous relations we deduce that

$$
\left|T_{i}(x)(t)-T_{i}(y)(t)\right| \leq \frac{d(x, y) e^{\tau(t-a)}}{\tau d(x, y)+1},
$$

hence

$$
\left|T_{i}(x)(t)-T_{i}(y)(t)\right| e^{-\tau(t-a)} \leq \frac{d(x, y)}{\tau d(x, y)+1},
$$


So

$$
\max _{i=\overline{1, n}} \max _{t \in[a, b]}\left(\left|T_{i}(x)(t)-T_{i}(y)(t)\right| e^{-\tau(t-a)}\right) \leq \frac{d(x, y)}{\tau d(x, y)+1}
$$

thus

$$
\|T(x)-T(y)\| \leq \frac{d(x, y)}{\tau d(x, y)+1}
$$

i.e.,

$$
d(T(x), T(y)) \leq \frac{d(x, y)}{\tau d(x, y)+1} .
$$

It follows that, for every $x, y \in C\left([a, b], \mathbb{R}^{n}\right), T(x) \neq T(y)$, we have

$$
\frac{\tau d(x, y)+1}{d(x, y)} \leq \frac{1}{d(T(x), T(y))},
$$

thus

$$
\tau-\frac{1}{d(T(x), T(y))} \leq-\frac{1}{d(x, y)}
$$

Considering the relation (19), we find

$$
\tau+F(d(T(x), T(y))) \leq F(d(x, y)), \text { for all } x, y \in C\left([a, b], \mathbb{R}^{n}\right), T(x) \neq T(y),
$$

hence the operator $T: C\left([a, b], \mathbb{R}^{n}\right) \rightarrow C\left([a, b], \mathbb{R}^{n}\right)$ is an $F$-contraction corresponding to the function class $\mathcal{F}^{\prime}$.

Moreover, from the relation (21) we deduce

$$
d(T(x), T(y))<\frac{1}{\tau}, \text { for every } x, y \in C\left([a, b], \mathbb{R}^{n}\right),
$$

thus there is a sequentially compact subset $S:=\left[0, \frac{1}{\tau}\right] \subseteq \mathbb{R}_{+}$having the property $\{d(T x, T y) \mid$ $x, y \in X\} \subseteq S$.

Consequently, the hypotheses of Corollary 1 are satisfied, hence the operator $T$ has a unique fixed point $x^{\star} \in C\left([a, b], \mathbb{R}^{n}\right)$. Further, for every $x_{0} \in C\left([a, b], \mathbb{R}^{n}\right)$ the sequence $\left(x_{n}\right)_{n \in \mathbb{N}} \subset C\left([a, b], \mathbb{R}^{n}\right)$, defined by $x_{n}=T x_{n-1}$ for all $n \in \mathbb{N}^{*}$, converges to $x^{\star}$. It follows that the integral Equation (16) has a unique solution in $C\left([a, b], \mathbb{R}^{n}\right)$.

In the sequel, we will apply Corollary 1 to a fractional integral equation. Following the study of Fernandez et al. [26] we consider $[a, b]$ a real interval, $\alpha \geq 0, \beta \geq 0, R>0$ satisfying $R>(b-a)^{\beta}$ and $A$ a real analytic function on the interval $(-R, R)$, defined by the locally uniformly convergent power series

$$
A(x)=\sum_{k=0}^{+\infty} a_{k}(\alpha, \beta) x^{k},
$$

where $a_{k}(\alpha, \beta), k \in \mathbb{N}$, are real coefficients.

Next, we determine some conditions for the existence and uniqueness of a solution of the following fractional integral equation

$$
x(t)=\int_{a}^{t}(t-s)^{\alpha+\gamma-1} A\left((t-s)^{\beta}\right) g(s, x(s)) d s+f(t), t \in[a, b],
$$

where $\gamma \geq 1, x:[a, b] \rightarrow \mathbb{R}, g:[a, b] \times \mathbb{R} \rightarrow \mathbb{R}, f:[a, b] \rightarrow \mathbb{R}$, by using Corollary 1 .

Considering the fractional integral operator ${ }^{A} I_{a+}^{\alpha, \beta}: L^{1}[a . b] \rightarrow L^{1}[a . b]$,

$$
{ }^{A} I_{a+g}^{\alpha, \beta} g(t):=\int_{a}^{t}(t-s)^{\alpha-1} A\left((t-s)^{\beta}\right) g(s) d s, t \in[a, b],
$$


defined by Fernandez et al. [26], the above fractional integral equation can be rewritten as

$$
x(t)={ }^{A} I_{a+}^{\alpha+\gamma, \beta} g(t, x(t))+f(t), t \in[a, b] .
$$

Theorem 3. If the functions $g:[a, b] \times \mathbb{R} \rightarrow \mathbb{R}, f:[a, b] \rightarrow \mathbb{R}$ are continuous and there is $\tau>0$ such that

$$
\left|(t-s)^{\alpha+\gamma-1} A\left((t-s)^{\beta}\right)(g(s, x(s))-g(s, y(s)))\right| \leq \frac{1}{b-a} \frac{|x(t)-y(t)|}{\tau \rho(x, y)+1},
$$

for all $t, s \in[a, b], s \leq t, x, y \in C([a, b], \mathbb{R})$, then the fractional integral Equation (23) has a unique solution in $C([a, b], \mathbb{R})$.

Proof. We consider $\left(\mathbb{R},+, \cdot, \tau_{\mathbb{R}}, \leq\right)$ the partially ordered Hausdorff topological ring and $\left(\mathbb{R},+, \cdot, \tau_{\mathbb{R}}\right)$ the Hausdorff topological left $\mathbb{R}$-module, where $\tau_{\mathbb{R}}$ is the Euclidean topology. Let $P=\mathbb{R}_{+}$be the solid cone, with $\operatorname{int}(P)=\mathbb{R}_{+}^{*}$. We define the set $C([a, b], \mathbb{R})=\{x$ : $[a, b] \rightarrow \mathbb{R} \mid x$ is continuous on $[a, b]\}$ and the mapping

$$
\rho: C([a, b], \mathbb{R}) \times C([a, b], \mathbb{R}) \rightarrow \mathbb{R}_{+}, \rho(x, y)=\max _{t \in[a, b]}\left(|x(t)-y(t)| e^{-\tau(t-a)}\right) .
$$

We remark that $(C([a, b], \mathbb{R}), \rho)$ is a complete cone metric space over the topological left $\mathbb{R}$-module. Moreover, the function

$$
F: \mathbb{R}_{+}^{*} \rightarrow \mathbb{R}, F(x)=-\frac{1}{x}
$$

belongs to the class $\mathcal{F}^{\prime}$.

As the functions $g:[a, b] \times \mathbb{R} \rightarrow \mathbb{R}$ and $f:[a, b] \rightarrow \mathbb{R}$ are continuous, we can define the operator $T: C([a, b], \mathbb{R}) \rightarrow C([a, b], \mathbb{R})$,

$$
T(x)(t)=\int_{a}^{t}(t-s)^{\alpha+\gamma-1} A\left((t-s)^{\beta}\right) g(s, x(s)) d s+f(t), t \in[a, b] .
$$

A function $x \in C([a, b], \mathbb{R})$ is a solution of the integral Equation (23) if and only if it is a fixed point of the operator $T$.

Considering the relation (26) we can easily prove that

$$
\rho(T(x), T(y)) \leq \frac{\rho(x, y)}{\tau \rho(x, y)+1}, \text { for all } x, y \in C([a, b], \mathbb{R}) .
$$

Therefore,

$$
\tau-\frac{1}{\rho(T(x), T(y))} \leq-\frac{1}{\rho(x, y)}, \text { for all } x, y \in C([a, b], \mathbb{R}), T(x) \neq T(y)
$$

and taking into account the relation (28) we deduce

$$
\tau+F(\rho(T(x), T(y))) \leq F(\rho(x, y)), \text { for all } x, y \in C([a, b], \mathbb{R}), T(x) \neq T(y),
$$

hence the operator $T: C([a, b], \mathbb{R}) \rightarrow C([a, b], \mathbb{R})$ is an $F$-contraction corresponding to the function class $\mathcal{F}^{\prime}$.

Moreover, from the relation (30) we obtain

$$
\rho(T(x), T(y))<\frac{1}{\tau}, \text { for every } x, y \in C([a, b], \mathbb{R}),
$$

thus there exists a sequentially compact subset $S:=\left[0, \frac{1}{\tau}\right] \subseteq \mathbb{R}_{+}$having the property $\{\rho(T x, T y) \mid x, y \in X\} \subseteq S$. 
Consequently, the hypotheses of Corollary 1 are satisfied, hence the operator $T$ has a unique fixed point $x^{\star} \in C([a, b], \mathbb{R})$. Further, for every $x_{0} \in C([a, b], \mathbb{R})$ the sequence $\left(x_{n}\right)_{n \in \mathbb{N}} \subset C([a, b], \mathbb{R})$, defined by $x_{n}=T x_{n-1}$ for all $n \in \mathbb{N}^{*}$, converges to $x^{\star}$. It follows that, the integral Equation (23) has a unique solution in $C([a, b], \mathbb{R})$.

Remark 2. A sufficient condition for fulfilling the inequality (26) is

$$
|g(s, x(s))-g(s, y(s))| \leq \frac{1}{(b-a)^{\alpha+\gamma} M} \frac{|x(t)-y(t)|}{\tau \rho(x, y)+1},
$$

for all $t, s \in[a, b], s \leq t, x, y \in C([a, b], \mathbb{R})$, where $M:=\sup _{x \in\left[0,(b-a)^{\beta}\right]}|A(x)|$.

Example 1. For $\beta=0, A(x)=\frac{1}{\Gamma(\alpha)}$, the fractional integral operator ${ }^{A} I_{a+}^{\alpha, \beta}$ becomes the original Riemann-Liouville fractional integral operator (RL integral operator)

$$
{ }^{R L} I_{a+g}^{\alpha} g(t):=\frac{1}{\Gamma(\alpha)} \int_{a}^{t}(t-s)^{\alpha-1} g(s) d s={ }^{A} I_{a+}^{\alpha, 0} g(t), t \in[a, b] .
$$

If the functions $g:[a, b] \times \mathbb{R} \rightarrow \mathbb{R}, f:[a, b] \rightarrow \mathbb{R}$ are continuous and there is $\tau>0$ such that

$$
\frac{1}{\Gamma(\alpha)}\left|(t-s)^{\alpha+\gamma-1}(g(s, x(s))-g(s, y(s)))\right| \leq \frac{1}{b-a} \frac{|x(t)-y(t)|}{\tau \rho(x, y)+1},
$$

for all $t, s \in[a, b], s \leq t, x, y \in C([a, b], \mathbb{R})$, then the fractional integral equation

$$
x(t)={ }^{R L} I_{a+}^{\alpha+\gamma} g(t, x(t))+f(t), t \in[a, b],
$$

has a unique solution in $C([a, b], \mathbb{R})$. A sufficient condition for fulfilling the inequality (33) is

$$
|g(s, x(s))-g(s, y(s))| \leq \frac{\Gamma(\alpha)}{(b-a)^{\alpha+\gamma}} \frac{|x(t)-y(t)|}{\tau \rho(x, y)+1},
$$

for all $t, s \in[a, b], s \leq t, x, y \in C([a, b], \mathbb{R})$.

Example 2. For $\beta=\delta \in(0,1], A(x)=\frac{1}{\Gamma(\alpha) \delta^{\alpha}} \exp \left(\frac{\delta-1}{\delta} x\right)$, the fractional integral operator ${ }^{A} I_{a+}^{\alpha, \beta}$ becomes the generalized proportional fractional integral operator (GPF integral operator)

$$
\begin{gathered}
G P F I_{a+\delta}^{\alpha, \delta} g(t):= \\
\frac{1}{\Gamma(\alpha) \delta^{\alpha}} \int_{a}^{t}(t-s)^{\alpha-1} \exp \left(\frac{\delta-1}{\delta}(t-s)\right) g(s) d s={ }^{A} I_{a+}^{\alpha, \delta} g(t), t \in[a, b] .
\end{gathered}
$$

If the functions $g:[a, b] \times \mathbb{R} \rightarrow \mathbb{R}, f:[a, b] \rightarrow \mathbb{R}$ are continuous and there is $\tau>0$ such that

$$
\begin{gathered}
\frac{1}{\Gamma(\alpha) \delta^{\alpha}}\left|(t-s)^{\alpha+\gamma-1} \exp \left(\frac{\delta-1}{\delta}(t-s)^{\delta}\right)(g(s, x(s))-g(s, y(s)))\right| \leq \\
\frac{1}{b-a} \frac{|x(t)-y(t)|}{\tau \rho(x, y)+1},
\end{gathered}
$$

for all $t, s \in[a, b], s \leq t, x, y \in C([a, b], \mathbb{R})$, then the fractional integral equation

$$
x(t)={ }^{G P F} I_{a+}^{\alpha+\gamma, \delta} g(t, x(t))+f(t), t \in[a, b],
$$


has a unique solution in $C([a, b], \mathbb{R})$. A sufficient condition for fulfilling the inequality (37) is

$$
|g(s, x(s))-g(s, y(s))| \leq \frac{\Gamma(\alpha) \delta^{\alpha}}{(b-a)^{\alpha+\gamma}} \frac{|x(t)-y(t)|}{\tau \rho(x, y)+1}
$$

for all $t, s \in[a, b], s \leq t, x, y \in C([a, b], \mathbb{R})$

\section{Conclusions}

In this paper, we define the notion of F-contraction for cone metric spaces over topological left modules and we establish some fixed point results for self-mappings satisfying a contractive condition of this type. Applications of the main result to the study of the existence and uniqueness of the solutions for certain types of integral equations were presented in the last part of the article, one of them being an integral equation of fractional type.

Funding: Project financed by Lucian Blaga University of Sibiu \& Hasso Plattner Foundation research grants LBUS-IRG-2021-07.

Data Availability Statement: Not applicable.

Acknowledgments: The author thanks the anonymous referees and the editors for their valuable comments and suggestions which improved greatly the quality of this paper.

Conflicts of Interest: The author declares no conflict of interest.

\section{References}

1. Huang, L.G.; Zhang, X. Cone metric spaces and fixed point theorems of contractive mappings. J. Math. Anal. Appl. 2007, 332, 1468-1476. [CrossRef]

2. Radenović, S.; Rhoades, B.E. Fixed point theorems for two non-self mappings in cone metric spaces. Comput. Math. Appl. 2009, 57, 1701-1707. [CrossRef]

3. Rezapour, S.; Hamlbarani, R. Some notes on the paper 'Cone metric spaces and fixed point theorems of contractive mappings'. J. Math. Anal. Appl. 2008, 345, 719-724. [CrossRef]

4. Kadelburg, Z.; Radenović, S.; Rakočević, V. A note on the equivalence of some metric and cone metric fixed point results. Appl. Math. Lett. 2011, 24, 370-374. [CrossRef]

5. Du, W.S. A note on cone metric fixed point theory and its equivalence. Nonlinear Anal. 2010, 72, 2259-2261. [CrossRef]

6. Liu, H.; Xu, S. Cone metric spaces with Banach algebras and fixed point theorems of generalized Lipschitz mappings. Fixed Point Theory Appl. 2013, 320, 1-10. [CrossRef]

7. Xu, S.; Radenović, S. Fixed point theorems of generalized Lipschitz mappings on cone metric spaces over Banach algebras without assumption of normality. Fixed Point Theory Appl. 2014, 102, 1-12. [CrossRef]

8. Wardowski, D. Fixed points of a new type of contractive mappings in complete metric spaces. Fixed Point Theory Appl. 2012, 94, 1-6. [CrossRef]

9. Wardowski, D.; Van Dung, N. Fixed points of F-weak contractions on complete metric space. Demonstr. Math. 2014, 47, 146-155. [CrossRef]

10. Cosentino, M.; Vetro, P. Fixed point results for F-contractive mappings of Hardy-Rogers-type. Filomat 2014, 28, 715-722. [CrossRef]

11. Piri, H.; Kumam, P. Some fixed point theorems concerning F-contraction in complete metric spaces. Fixed Point Theory Appl. 2014, 210, 1-11. [CrossRef]

12. Minak, N.G.; Helvaci, A.; Altun, I. Cirić type generalized F-contractions on complete metric spaces and fixed point results. Filomat 2014, 28, 1143-1151. [CrossRef]

13. Ahmad, J.; Al-Rawashdeh, A.; Azam A. New fixed point theorems for generalized F-contractions in complete metric spaces. Fixed Point Theory Appl. 2015, 80, 1-18. [CrossRef]

14. Kadelburg, Z.; Radenović, S. Notes on some recent papers concerning F-contractions in b-metric spaces. Constr. Math. Anal. 2018, 1, 108-112. [CrossRef]

15. Dey, L.K.; Kumam, P.; Senapati, T. Fixed point results concerning F-contraction mappings in metric spaces. Appl. Gen. Topol. 2019, 20, 81-95. [CrossRef]

16. Wardowski, D. Solving existence problems via F-contractions. Proc. Am. Math. Soc. 2018, 146, 1585-1598. [CrossRef]

17. Alfaqih, W.M.; Imdad, M.; Gubran, R. An observation on F-weak contractions and discontinuity at the fixed point with an application. J. Fixed Point Theory Appl. 2020, 22, 1-10. [CrossRef]

18. Karapinar, E.; Fulga, A.; Agarwal, R.P. A survey: F-contractions with related fixed point results. J. Fixed Point Theory Appl. 2020, 22, 1-58. [CrossRef]

19. Liouville, J. Mémoire sur quelques questions de géometrie et de mécanique, et sur un nouveau genre de calcul pour résoudre ces questions. J. Polytech. Math. 1832, 13, 1-69. 
20. Caputo, M. Linear models of dissipation whose $q$ is almost frequency independent-II. Geophys. Int. J. 1967, 13, 529-539. [CrossRef]

21. Atangana, A.; Baleanu, D. New fractional derivatives with nonlocal and non-singular kernel: Theory and application to heat transfer model. Therm. Sci. 2016, 20, 763-769. [CrossRef]

22. Baleanu, D.; Fernez, A. On some new properties of fractional derivatives with Mittag-Leffler kernel. Commun. Nonlinear Sci. Numer. Simul. 2018, 59, 444-462. [CrossRef]

23. Jarad, F.; Abdeljawad, T.; Alzabut, J. Generalized fractional derivatives generated by a class of local proportional derivatives. Eur. Phys. J. Spec. Top. 2018, 226, 3457-3471. [CrossRef]

24. Prabhakar, T.R. A singular integral equation with a generalized Mittag Leffler function in the kernel. Yokohama Math. J. 1971, 19, 7-15.

25. Kilbas, A.A.; Saigo, M.; Saxena, R.K. Generalized Mittag-Leffler function and generalized fractional calculus operators. Integr. Transf. Spec. Funct. 2004, 15, 31-49. [CrossRef]

26. Fernandez, A.; Özarslan, M.A.; Baleanu, D. On fractional calculus with general analytic kernels. Appl. Math. Comput. 2019, 354, 248-265. [CrossRef]

27. Agarwal, R.P.; Benchohra, M.; Hamani, S. A survey on existence results for boundary value problems of nonlinear fractional differential equations and inclusions. Acta Appl. Math. 2010, 109, 973-1033. [CrossRef]

28. Agarwal, R.P.; Hristova, S.; O'Regan, D.; Almeida, R. Approximate iterative method for initial value problem of impulsive fractional differential equations with generalized proportional fractional derivatives. Mathematics 2021, 9, 1979. [CrossRef]

29. Soradi-Zeid, S.; Mesrizadeh, M.; Cattani, C. Numerical solutions of fractional differential equations by using Laplace transformation method and quadrature rule. Fractal Fract. 2021, 5, 111. [CrossRef]

30. Almeida, R. Functional differential equations involving the $\Psi$-Caputo fractional derivative. Fractal Fract. 2020, 4, 29. [CrossRef]

31. Khan, H.; Shah, R.; Kumam, P.; Baleanu, D.; Arif, M. An efficient analytical technique for the solution of fractional-order telegraph equations. Mathematics 2019, 7, 426. [CrossRef]

32. Keten, A.; Yavuz, M.; Baleanu, D. Nonlocal Cauchy problem via a fractional operator involving power kernel in Banach spaces. Fractal Fract. 2019, 3, 27. [CrossRef]

33. Warner, S. Topological Rings; North-Holland Mathematics Studies; Elsevier: Amsterdam, The Netherlands, 1993. 